\title{
Ueber die ungleiche Entfexnung von Doppelbildern, welche in verschiedener Höhe gesehen werden.
}

\author{
Von
}

Dr. Albrecht Nagel.

\begin{abstract}
$\mathrm{E}_{\mathrm{s}}$ ist in diesem Archiv wiederholt zur Sprache gekommen, dass Doppelbilder, welche in verschiedener Höhe gesehen werden, in unglciches Entfernung vom Beobachter sich zu befinden scheinen. Diese Beobachtung wurde zuerst in pathologischen Fällen gemacht. Namentlich war es das bei Lähmung des oberen schiefen Augenmuskels beobachtete Näherstehen des tieferen Doppelbildes, welches die Aufmerksamkeit der Pathologen auf sich zog. v. Gra efe ${ }^{*}$ ) giebt bei Besprechung der Symptomengruppe der 'Trochlearislähnung eine sehr treue Schilderung der Lage der Loppcibilder, nach den Angaben seiner Kranken. Doppeltschen tritt vorzugsweise ein, wenn der Kranke seinen Blick mach unten richtet. Alsdann tritt neben dem init dem gesundén Auge an richtigen Orte gesehenen Bilde eines fixirten Objects ein. Doppelbild

*) Archir f. Ophthalm. Bd. I. Abth. 1, pag. 68.
\end{abstract}


desselben auf, welches etwas seitwärts, auf Seite des kranken Auges, erheblich tiefer steht und eine deutliche Schiefheit, durch Yeigung nach innen zeigt. Ziugleich soll dies Doppelbild deutlich näher stehen und etwas grösser erscheinen, als das anlere mit dem gesunden Auge gesehene Doppelbild. Iine Frrklärung dieser tiplopischon Frscheinungen, welche von der v. Graefe'schen Erklärung in einem Hauptpunkt abweicht, habe ich in meinem Buche: „Das Sehen mit zwei Augen und die Lehre von den identischen Netzhiutstellen, Leipzig und IIeidelberg 1861" gegeben*) und will dicselbe hier nicht wiederholen. Niur das Näherstehen des Doppelbildes soll, da es von besonderem physiologischem Interesse ist, den Gegenstand einiger Betrachtmugen bilden.

Ich gehe anf dieses Thona mit einiger Ausführlichkeit cin, weil ich es als einen l'rüfstein ansehe für die Richtigkeit der von mir an beceichneten Orte aufgestellten Auffissung sämmtlicher Eirscheinungen des Doppeltsehens. - v. Crraefo sagt zur Erläuterung der auffalIenden Erscheinung: „Das scheiubare Näherstchen wird durch die pathologische Convergenz und den engeren Ioropter bedingt.:" Ausser diesem etwas dunkeln Ausspruch ist mir eine weitere Lirwahnung dieses Punktes in v. Graefe's Schriften nicht bekannt. Ausführlicheres findet sich jedoch bei Alfred (iraefe.*) Dieser theilt mit, dass bei l'rochlearislähmung constant die Walırnehinungr gemacht werle, dass das Bild des kranken Auges bei allen Lagen des Objects immer näher stehe, als das des gesunden. Inurch einen Druck, welcher abwechselnd auf das kranke und auf das gesunde Auge in der Richtung von yorn llach hinten ausgeübt werde, könne das Doppelbild des erkrankten Auges noch näher gebracht

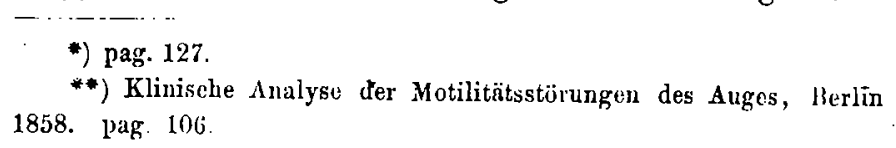

**) Klinische Analyse đer Motilitätsstörungen des Auges, Berlin 1858. pag 106 . 
resp. mit dem Bilde des gesunden Auges in gleichen $\mathrm{Ab}$ stand verlegt werden (?). Anfänglich habe v. Graefe das Phänomen aus einer Verrückung des Drehpunktes des Auges nach hinten erklären wollen, wie er einell solchen beim $\Lambda$ ufhören der nach vorne ziehenden Wirkung des M. obliquus superior erwarten zu müssen glaubte, zumal dies durch jene Experimente mit künstlicher Dislocation des Augendrehpunktes bestätigt zu werden schiene. Doch habe v. Graefe diese Erklärung wieder verlassen, da sie ihm selbst nicht genügt habe.*) Eben deshalb kann ich mich in der Beleuchtung dieser Ansicht kurz fassen. - „Der Drehpunkt des Auges muss theoretisch bei der Lähmung des Trochlcaris etwas nach hinten verrückt sein," sagt A. v. Graefe. Weshalb? deutet A. Graefe näher an: "Die auf den Bulbus retrahirend wirkenden geraden A ugenmuskeln wurden durch Lähmung des Trochlearis eines Theils ihrer antagonistischen Gegenwirkung beraubt." Dass die beiden Obliqui zusanmen als Antagonisten der Recti betrachtet werden, ist gewiss nur im Allgemeinen und Ungefähren gemeint worden, und ob sie wirklich jene antagonistische Wirkung des Nachvornziehens ausüben, ist durchaus nicht erwiesen. Theoretisch wäre es denkbar, dass beide Obliqui bei geneinsamer Action neben anderem Einfluss auf die Stellung des Bulbus dessen Drehpunkt um ein Weniges nach vorne (und stark nach innen) zu dislociren vermöchten, allein abgesehen davon, dass andere Gründe dies unwahrscheinlich machen, scheinen mir gerade die Erscheinungen bei Paralyse des M. Obliquus superior nicht dafür zu sprechen. Bliebe nämlich die Wirkung des einen Obliquus aus, so müsste der Bulbus um so leichter dem gleichzeitig wirkenden Zuge des anderen Obliquus folgen, es müssten gleich von vorn herein bei frischer Troch-

*) cf. A. Graefe im Archir f. Ophth. Bd. VII. Abth. 2 pag. 109. 
learislähnung dieselben Symptome auftreten, wie sie in Wirklichkeit erst im weiteren Verlauf nach längerer Dauer der Paralyse stattfinden, nämlich die Symptome der Contractur des Obliquus inferior, schielende Stellung bei fast allen Augenrichtungen und über das ganze Gesichtsfeld rerbreitetes I)oppeltsehen mit ,charakteristisch geneigten boppelbildern. Mir ist es viel wahrscheinlicher, dass die Analogie mit dem Exophthalmos paralyticus bei Oculomotoriuslähmung keine zutreffende ist, und dass eine Veränderung in der Lage des Augendrehpunktes in der Orbita bci gewöhnlicher einfacher Trochlearislähmung nicht stattfindet. Dies bestätigen übrigens auch die eigenen Beobachtungen v. Graefe's, der sich auch hier als der gewissenhafte Beobachter beweist, dessen Objectivität unter der Theorie nicht leidet. In der Regel wurde von ihm ein Zurücktreten des Bulbus bei Trochlearislähniung nicht wahrgenommen.

Uebrigens würde, auch wenn eine Veränderung in der Lage des Augendrehpunktes festgestellt wäre, diesclbe zur Erklärung des Näherstehens gewisser Doppelbilder noch keineswegs genügen. Die Entfernung des Doppelbildes ist ja überhaupt nicht abhängig von der Function eines Muskels, sondern zunächst nur die Richtung, in welcher dasselbe gesehen wird. Auch ist es bekannt, dass mit dem oft sehr starken Hervortreten des Bulbus bei Oculomotoriuslähmung ein weiteres Abstehen des Doppelbildes, wie man solches der Analogie nach erwarten musste, keineswegs Statt hat. Ich gehe indessen auf diesen Gegenstand nicht weiter ein, da v. Graefe selbst seine aus der Veränderung des Drehpunktes entnommene Erklärung für das Näherstehen der tieferen Doppelbilder nicht mehr aufrecht erhält.

Die Frage war also eine offene; es musste nach einer neuen, den Thatsachen sich besser anschliessenden Erkläruug gesucht werden. Eine solche stellte Foerster 
auf und ihm schloss sich A. Graefe an. Beide suchen den ungleichen Abstand der in verschiedener Höhe gesehenen Doppelbilder bei Trochlearislähmung durch analoge physiologische Erscheinungen zu erklären, so dass jenes Verhạlten nicht als etwas spezifisch Pathologisches betrachtet werden müsse, das nur der Erkrankung jenes Muskels eigenthümlich sei, sondern als eine Erscheinung, die sich jedesmal wiederhole, wenn aus irgend einem Grunde Doppelbilder in verschiedener Höhe erscheinen. Wenn ich auch hierin mit beiden Autoren vollkommen übereinstimme, so doch nicht in dem Detail der Erklärung.

Foerster's Ansicht ist folgende: „Wenn wir einen Gegenstand, der sich auf einer horizontalen Fläche befindet, (schräg von oben herab) ansehen, so wird sein Bild auf die Macula lutea zu liegen kommen. Alles, was sich auf dieser Fläche $z$ wischen uns und dem fixirten Punkte befindet, bildet sich anf der oberen Hälfte, was jenseits des fixirten Punktes liegt, auf der unteren Iälfte der Netzhaut ab. Wir sind daher gewöhnt, den Gegenstand für näher zu halten, der sich oberhalb der Macula lutea abbildet."*)

So folgert Foerster und wendet dies auf die Lähmung des M. obliq. superior an. Da beim Blick nach unten das so erkrankte Auge nach oben und ein wenig nach innen von der Fixationsrichtung abweicht, so wird das Bild des fixirten Punktes auf die obere Hälfte der Netzhaut fallen; aus dem eben erwähnten Grunde soll das Doppelbild des erkrankten Auges näher als das Bild des gesunden Auges zu stehen scheinen. Aber auch bei gesunden Augen soll sich jene Regel bewahrheiten, wenn nämlich durch prismatische Ablenkung oder, wie A. Gra efe

*) Ueber das Näherstehen der tieferen Doppilbilder bei Lähmung des Musc. obliquus superior im 37. Jahresbericht der schles. Gesellschaft für vaterländische Cultur. 1859. pag. 11 
ergänt, durch mechanisches Verschieben eines Bulbus Joppelbilder von verschiedenem Höhenstande künstlich crzeugt werden.

The ich zur experimentellen Prüfung der behaupteten 'Thatsache und zur Kritik der F'oerster'schen Erklärung derselben übergehe, will ich mit wenigen Worten die IIauptsätze hervorheben, welche nach meiner Auflassung der Deutung aller Krscheinungen des Doppeltsehens zu Grunde gelegt werden müssen.*)

Man muss zwei Gattungen von Doppeltsehen unterscheiden, einmal dasjenige, welches excentrisch gelegene Schobjecte bei einfachem Frscheinen eines beiderseitig fixirten Objects betrifft, sodaun dasjenige, bei dem das zu fixirende Object und mit diesem das ganze Gesichtsfuld des einen Auges verschoben erscheint. Das erstere Ioppeltsehen beruht auf falscher Auffassung der Entfernung; letzteres auf falscher Auffassung der Richtung. Nur die zweite Gattung des Doppeltsehens beschäftigt uns hier und auf dieses beschränke ich meine allgemeinen Erörterungen.

Gleichgültig ob auf natürlichem Wege durch Erkrankung im oculomotorischen Apparat, ob auf künstlichem Wege durch mechanische Verschiebung eimes Bulbus oder lurch prismatische Ablenkung des einfallenden Lichts die Cnmöglichkeit gegeben ist, die Lage des eigenen Auges richtig $\mathrm{zu}$ beurtheilen und somit las empfangene Bild in angemessener Richtung in die Aussenwelt zu verlegen, so hat in allen Fällen die zweite Gattung des Doppeltsehens die Eigenthümlichkeit, dass die Wahrnehmung der Fntfernung dabei nur secundär gestört ist. Im Gegensatz dazu nenne ich es zum Beispiel eine primäre Störung der Wahrnehmung der richtigen Fntfernung, wenn von zwei hinter cinander gehaltenen Fingern abwechselnd der

*) ef. mein oben erwähntes Buch, fïnftes Kapitel. 
eine fixirt wird. Der nicht fixirte Finger erscheint dann doppelt in Folge davon, dass seine Entfernung unrichtig aufgefasst wird. Anders in den erwähnten Fällen, wo das Netzhautbild in falscher Richtung nach aussen projicirt wird und wo dann auch die Schätzung der Entfernung secundär lediglich desshalb beeinträchtigt wird, weil nun die beiden Netzhautbilder nicht zur Vereinigung und gegenseitigen Rectification betreffs der Abstände der einzelnen Bildpunkte benutzt werden, sondern jedes Auge gewissermaassen für sich und unabhängig von seinem Genossen sieht. Genaue und richtige Schätzung der Entfernung, wenigstens der relativen Entfernung jedes einzelnen Punktes und somit Wahrnehmung der Körperlichkeit des Objects ist das wichtigste Resultat der gemeinsamen Thätiglseit beider Augen. Das Resultat bleibt aus, wenn beide Augen nicht in normaler Weise zusammen wirken. Bei jedem Doppeltsehen ohne Unterschied wird man also Verminderung des körperlichen Eindrucks beobachten, es besteht namentlich bei nicht ganz bekannten Gesichtsobjecten die Neigung, alle Punkte des Objects in gleiche Entfernung, also in eine sphärische Fläche zu verlegen. Wie weit das ganze Sehobject resp. Doppelbild nach aussen zu verlegen sei, welcher Radius der Projectionssphäre zu geben sei, das hängt von den Bedingungen $a b$, die bei monocularem Sehen überhaupt gelten. Dieselben Mittel, welche wir zur Taxation der Entfernung beim Sehen mit einem Auge haben, stehen uns auch beim Doppeltsehen mit zwei Augen zu Gebote. Natürlich gelten dann die, die Wahrnehmung der Entfernung beeinflussenden Momente, im Allgemeinen für beide Doppelbilder in gleicher Weise. Die Doppelbilder beider Augen werden in der Regel, und wenn nicht besondere Gründe eine Aenderung bedingen, in gleiche Entfernung verlegt. Sphärische Flächen von gleichem Radius, deren Mittelpunkt die Kreuzungspunkte 
der Projectionslinie*) in beiden $A$ ugen sind, dienen im Allgemeinen zur Localisation der Doppelbilder.

Bei der Besprechung einiger Experimente wird sich Gelegenheit bieten, diese allgemeinen Regeln anzuwenden und zu erläutern. Am geeignetsten zum Experimentiren sind die durch prismatische Gläser erzeugten Doppelbilder.

Versuch I. Man halte ein Prisma mit der Basis nach oben vor ein Auge und blicke mit beiden Augen auf einen etwas tiefer befindlichen Gegenstand herab, so dass der Blick schräg abwärts gerichtet ist. Eine 'Tischecke bilde das Fixirobject. Man sieht dieselbe in über einander befindlichen Doppelbildern und bemerkt, dass das untere durch das Prisma gesehene Doppelbild ein wenig näher zu liegen scheint. Vertrïgt sich das mit obiger Annahrne, dass beide Doppelbilder in gleichen Abstand verlegt werden? Ganz gewiss. Man denke sich zwei Kugelfächen durch den fixirten Punkt gelegt, deren Mittelpunkte die Kreuzungspunkte der Visirlinien beider Augen sind, oder einfacher, man denke sich diese beiden l'rojectionssphären, die bei grossem Radius nahezu zusammen fallen, in eine vereinigt und betrachte den in der Medianebene beider Augen liegenden, also durch den Fixirpunkt gehenden grössten Kreis. Wiewohl jeder Punkt desselben von dem Auge natürlich gleich weit entfernt ist, so sind doch dem Körper des Beobachters die Punkte um so näher, je weiter nach oben oder nach unten sie liegen, je weiter sie sich von dem horizontalen Durchmesser des Kreises entfernen. Bezieht man also bei Schätzung der Entfernung der doppelt gesehenen Tischecke das Maas auf den verticalen Abstand von dem aufrecht stehenden Körper, so hat es mit obiger Beobach-

*) Projectionslinie oder Visirlinie nenno ich diejenige Gerade, welche den Objectpunkt mit seinem Netzhautbilde verbindet. 
tung seine Richtigkeit; dieselbe bedarf keiner weiteren Erklärung. In der. That, sieht man das Resultat des Experiments darauf an, so wird man leicht wahrnehmen, dass man wirklich in der bezeichneten Weise den $A b$ stand der Doppelbilder abschätzt. Man nennt die Punkte gleich weit abstehend, welche in einer der Körperaxe parallelen Ebene liegen. Achtet man im obigen Falle auf den directen Abstand beider Doppelbilder vom Auge, so wird man einen Unterschied nicht constatiren können; vieimehr stimmt der Unterschied in der Entfernung der Doppelbilder, den man anfangs zu bemerken glaubte, ganz mit der Localisation der Bilder in die Projectionssphäre überein. Doch eine Bestätigung wird willkommen sein. Man.lege eine Kugel auf die Tischecke. Deren Grösse in beiden Doppelbildern wird Aufschluss geben, ob beide Bilder in gleiche Entfernung projicirt worden oder nicht. Da nun beim Blick durchs Prisma wie oben die Bilder beider Kugeln genau gleich gross erscheinen, so haben wir einen lieweis, dass die Projectionssphären beider Augen gleichen Radius haben, - wofern nicht andere Versuche //weifel dagegen erwecken.

Versuch II. Die Kugel wird mitten auf die horizontale Tischplatte gelegt und wie vorhin betrachtet, während das eine Auge nit dem das Licht nach oben ablenkendeu Prisma versehen ist. Das Resultat ist jetzt entschieden ein anderes, das untere Doppelbild erscheint viel näher als das obere, auch ist es unzweifelhaft kleiner. Wird das Prisma umgekehrt, so dass die Basis nach unten sieht, so erscheinen die Doppelbilder höher als zuvor, aber der Unterschied in Betreff der Entfernung und Grösse ist der nämliche, das tiefere Doppelbild ist das nähere, kleinere. Der Unterschied in dem Abstande der Bilder ist nunmehr so bedeutend, dass er nicht mehr wie in Versuch I. erklärt werden kann, auch zeigt der Grössenunterschied der Doppelbilder deutlich genug, dass 
sie in verschiedene Ferue projicirt werden, demu die Netzhautbilder haben ja in beiden Augen dieselbe Grösse. Bei öfterer Wiederholung und längerer Dauer des Experiments wird man dem Grunde des abweichenden Resultates auf die Spur kommen. Man gewahrt dann, dass die beschriebene Erscheinung kcinc constante ist. Von Zeit zll Zeit tritt wenigstens für Augenblicke das näher stehende Doppelbild zurïck und wird an Grösse dem andern gleich, viel häufiger freilich erscheint das tiefere Bild näher und kleiner. Yan bemerkt dann, das in dem durch das Prisma gesehenen Bilde die Tischplatte gewissermaassen in der Verlängerung der wirklichen Tischplatte nach dem Beobachter zu zu liegen scheint und auf dieser verlängerten 'Tischplatte sieht man das Doppelbild der Kugel liegen. Es ist klar, dass die Kugel alsdanu eine viel weniger tiefe und zugleich einc viel nähere Stellung cinnehmen muss, als in dem früher erwähnten Falle (Versuch I.). Die natürlichen, gewohnheitsgemässen Verhältnisse des Sehens, die natürliche Neigung, ähnliche Bilder beider Augen zur einheitlichen Verschmelzung zu bringen, liegt hicr offenbar zu Grunde. Eine völlige Verschmelzung der von beiden Augen gesehenen 'I'ischplatten ist nicht möglich, weil die Grenzen beider in zu verschiedener Richtung gesehen werden, aber es decken sich die beiden Platten wenigstens theilweise und dann werden die übrigen Theile in die gleiche Fbene, der eine in die Verlängerung des anderen gebracht. Dann ist es auch erklärlich, wie das Doppelbild der Kugel in gleicher Weise dislocirt erscheint. Ein Widerspruch gegen die oben aufgestellte allgemeine Regel liegt darin nicht. Die Verlegung der Doppelbilder in die Projectionssphären ist ja nur ein Nothbehelf, der dann eintritt, wenn kein anderer Anhaltspunkt für die Localisation da ist. Jedes Moment, welches, sei es berichtigend, sei es irreleitend, beim Doppeltsehen die Vorstellung von der Entfernung 
beeinflusst, hebt dadurch die Verlegung in die Projectionssphären auf.

Als Beweis dafür, dass im Versuch II. wirklich das durch das Prisma gesehene Doppelbild auf die horizontale Ebene projicirt wird, wenn ein solcher noch nöthig ist und nicht vielmehr der Augenschein schon genügt, diene die Beobachtung, dass der Abstand des Doppelbildes von den wahren (d. h. mit freiem Auge gesehenen) Bilde, auf der horizontalen Fläche gemcssen, verschieden zu sein scheint, je nachdem die brechende Kante des P'rismas nach oben oder unten gerichtet ist, oder, was dasselbe ist, je nachdem das durch das Prisma gesehene Doppelbild eine höhere oder tiefere Stellung inne hat. Wie sich aus beistehender Figur ergiebt, wo $A$ das Auge, $\boldsymbol{K}, \boldsymbol{h}_{n}$, die Tischplatte, $\boldsymbol{K}$ das wahre Bild, $\boldsymbol{K}$, und $\boldsymbol{K}_{n}$, die Doppelbilder in jenen beiden Fällen bedeuten, ist es ein bloss perspectivischer Grund, weshalb im ersten Falle jener Abstand grösser zu sein scheint; denn da $<K A h$, $=<K A K_{n}$ ist, so muss $K K_{n}$ grösser sein als $K K_{r}$.

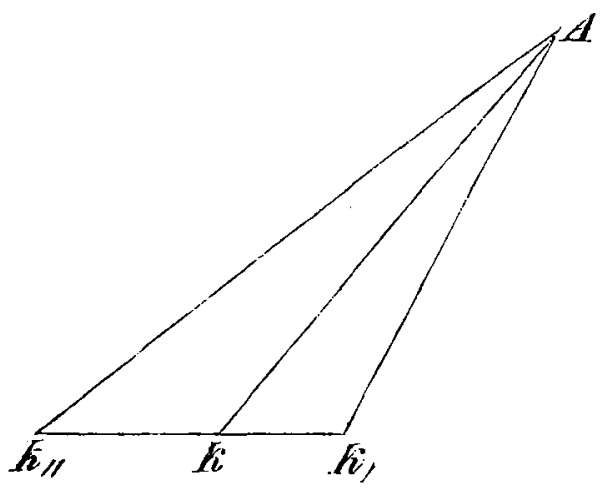

Wer noch Zweifel in die Richtigkeit der gegebenen Intergretation der Differenz in der lintfernung und Grösse der Doppelbilder setzt, beobachte bei Anstellung des Versuchs Entfernung und Grösse der umgebenden Gegen- 
stände in dem durch das Prisma verschobenen Gesichtsfelde. Man wird dann erkennen, dass nicht etwa alle Gegenstände, welche tief gesehen werden, zugleich näher und kleiner erscheinen. Da sich im Allgemeinen bei andern Objecten dieser Unterschied nicht kund giebt, so muss die Erscheinung in dem einen Falle einen besonderen, ausnahmsweisen Grund haben, wie ich ihn oben klar gelegt zu haben glaube.

Fis wird nun darauf ankommen, durch Modification des Fxperiments die Richtigkeit der Auffassung zu prüfen. Ich untersuchte das Verhalten der Doppelbilder von Gegenständen, welche sich oberhalb der horizontaleı befanden, zu deren Fixirung also das Auge schräg in die Höhe gerichtet werden musste.

Versuch III. Wenn ich unter Application eines Prismas mit der Basis nach oben vor ein Auge die vorspringende obere Ecke eines Schrankes von der Seite betrachtete, so dass eine Wand den Hintergrund bildete, auf dem die Doppelbilder erscheinen, so stellte sich gerade das umgekehrte Verhalten der Doppelbilder heraus wie in Versuch I. Das tiefere Doppelbild war jetzt deutlich entfernter als das höhere, wenn man nämlich die scheinbaren senkrechten Abstände von der Körperaxe des aufrecht stehenden Beobachters verglich. Die Stellung der Doppelbilder war übrigens derart, dass sie gänzlich mit der bei Versuch I. geltend gemachten Auffassung übereinstimmte. Vom Auge aus schienen sie gleich weit entfernt, in gleicher Projectionssphäre zu liegen. Eine am Fixirpunkt angebrachte Kugel lieferte hierfür insofern eine Bestätigung, als die Doppelbilder genau die gleiche Grösse hatten.

Versuch IV. Wenn ich nun dieselbe Kugel an die Decke des Zimmers befestigte und wie in Versuch III. betrachtete, so erschien die Lage des Doppelbildes wiederum wesentlich verändert. Es trat viel weiter zurück und 
schien an der Zimmerdecke befestigt zu sein, ein Resultat, welches der Analogie mit Versuch II. zufolge erwartet werden musste. Auch erschien das Doppelbild deutlich vergrössert, um so ferner und grösser, je grösser die Höhe der Zimmerdecke und je stärkar das ablenkende Prisma war. Die Frklärung ist einfach. Die Doppelbilder der Zimmerdecke markiren sich durch Begrenzung so wenig, dass sic zur theilweisen Deckung gebracht werden und das falsche Bild der Kugel wird wie das wahre auf die zusammenhängende ebone Fläche projicirt. Auch hier crlcidet die Verlegung in die Projectionssphäre eine Störung durch die Intervention des nach Gewohnheitsgesetzen localisirenden Verstandes.

Es war mir nun wünschenswerth, diese Verstandesthätigkeit in anderen Fällen wo möglich in abweichender Richtıng thätig zu sehen. Für ein cinzeln dastehendes Phänomen lassen sich ja leicht noch andere Erklärungen finden; erst die Uebereinstimmung in verschiedenen Fällen, der Zusammenhang der Thatsachen vermag die Wahrscheinlichkeit zur Gewissheit, die Vermuthung zur Ueberzeugung zu erheben.

Während es in Versuch II. und IV. eine horizontale Fläche war, welche die normgemässe Projection alterirte, so lag es uahe, zu prüfen, ob eine verticale Fläche oder Linie ähnliche Wirkung äussert.

Versuch V. Zu diesem Zwecke hängte ich eine Kugcl an einem Faden frei auf und erzeugte durch Bewaffnung des einen Auges mit einem Prisma Doppeltsehen mit vertical distanten Doppelbildern. Wurde die Kugel tief gehängt, so dass ich in stehender Stellung auf dieselbe herabsah, so zeigte sich sogleich in der Lage der in verschiedener Höhe gesehenen Doppelbilder eine Verschiedenheit gegen die Versucho I. und II. Das tiefere 1)oppelbild befand sich (gleichgültig ob die Basis des Prismas nach oben oder unten gekehrt \%ar), wenn keine 
seitliche Differenz in der Lage beider Bilder bestand, mit dem es tragenden Faden in der Verlängerung des mit dem anderen Auge direkt gesehenen Fadens. Die untere Kugel schien vertical unter der oberen zu schweben, beide an demselben Faden befestigt zu sein. Der verticale Abstand beider Kugeln von dem Körper des Beobachters schien also derselbe zu sein, der gerade Abstand vom Auge war für das untere Doppelbild entschieden grösser, ganz im Gegensatz zu Versuch II., wo das ticfere Doppelbild das nähere war. Auch war das untere Doppelbild in diesem Versuche das grössere, während es in Versuch II. das klcinere war. 7ur Controle der Projectionsweise dient bei diesern Versuche wieder der Umstand, dass der Abstand des künstlich erzeugten Doppelbildes ron dem wahren bilde verschieden gross ist, je nachdem durch die Stel-

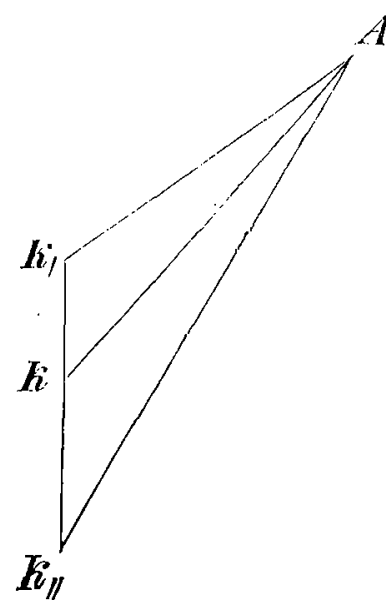

lung des Prisinas das Doppelbild obcrhalb oder unterhalb des wahren Bildes gesehen wird. In beistehender Figur bezeichnet $A$ das Auge, $K, K_{n}$ die Richtung des hängenden Fadens mit dem wahren Bilde $K$ und den beiden Doppelbildern $K$, und $k_{\prime \prime}$. Da $<\boldsymbol{K} \boldsymbol{A} \boldsymbol{K}_{,}=<\boldsymbol{K} \boldsymbol{A} \boldsymbol{A} \boldsymbol{K}_{\prime \prime}$ ist, so muss $K K_{\prime \prime}$ grösser sein als $\boldsymbol{K} \boldsymbol{K}_{\boldsymbol{\prime}}$.

\%u bemerken ist übrigens, dass auch boi diesem Versuche das Resultat keineswegs immer constant ist. Es wechseln rerschiedene Projectionsweisen mit einander $a b$, ja sie känpfen mit einander. Es treten bisweilen Momente ein, wo beide Doppelbilder in gleicher Entfernung und von gleicher Grösse erscheinen, wo sie also in Projectionssphären von gleichem Radius verlegt 
werden. Alsdann ist es auch auffällig, wie die Doppelbilder des Fadens oft nicht gerade, sondern gekrümmt erscheinen, in Richtung und Stärke der Krümmung gan?. der Projectionssphäre entsprechend, die Concavität der Krümmung dem betreffenden Auge zukehrend - eine Frscheinung, die ich bei einem ähnlichen Versuche bereits erwähnt habe*) und auf deren Beweiskraft für die Localisation der Doppelbilder in eine sphärisch gekrümmte l'läche ich hier nochmals im Vorbeigehen aufmerksam mache.

Ueber solche kleinen Variationen in der Lage, Grösse und Gestalt der Doppelbilder können wir uns nicht wundern, nachdem wir die Bedingungen kennen gelernt haben, unter denen dieselben vorkommen, und die Ursachen, welche ihnen zu Grunde liegen.

Es gelang mir nun, die bisherigen Versuche gewissermaassen in einen zu combiniren, dessen Instruktivität und schlagende Beweiskraft für die aufgestellten Ansichten nichts zu wünschen übrig liess.

Versuch VI. Von zwei gleichen Kugeln legte ich die eine auf den Rand eines Tisches, die andere hängte ich vermittelst eines Fadens an die Zimmerdecke in der Weise auf, dass beide Kugeln sich, wenige Zolle von cinander entfernt, in genau gleicher Höhe befanden. Während ich nun bei aufrechter Körperstellung auf beide neben einander befindlichen Kugeln hinabsah, hielt ich ein Prisma von 18 Grad vor ein Auge, die brechende Kante abwärts gerichtet. Jede Kugel wurde nun doppelt gesehen, aber merkwürdig! - die Lage der beiden tieferen Doppelbilder, die man als giluz gleich hätte erwarten mögen, war ganz verschieden. Das durch das Prisma gesehene tiefere Doppelbild der auf dem Tische liegenden Kugel schien $n$ äher als das wahre (mit freiem Auge

*) cf. meine oben erwähnte Schrift pag. 108. 
gesehene) Bild, das Doppelbild der hängenden Kugel schien ferner als das zugehörige wahre Bild. Die beiden Doppelbilder befanden sich also in verschiedener Ferne. und in verschiedener Höhc. Auch deutliche Grössenunterschiede fanden statt. Das Doppelbild der liegenden Kugel war kleiner, das der hängenden Kugel hingegen etwas grösser als die direct geschenen Kugeln. Die $\mathrm{U}_{1-}$ sache dieser Verschiedenheit ist aus dem Vorhergehenden klar. Das Doppelbild der liegenden Kugel wurde auf die Verlängerung der 'Tischplatte projicirt, das Doppelbild der hängenden Kugel mit dem sie tragenden Faden in die Verlängerung dieses letzteren. Bei wiederholtem Anschauen bemerkt man manche Aenderungen. Zuweilen sicht man beide tiefere Kugeln vorne in gleichem $\mathrm{Ab}$ stande, wo vorher nur die liegende gesehen wurde, dies namentlich beim crsten Hinblicken und wenn man auf den Aufhängungsfaden nicht Acht giebt oder seine deutliche Wahrnehmung durch ein optisches Hülfsmittel, etwa durch ein Convexglas, verhindert. Richtet man dann jedoch die Aufmerksamkeit auf den Faden, so verlässt das betreffende Doppelbild plïtzlich seinen Platz, um sich lothrecht unter das direkt gesehene Bild in die Verlängerung des Fadens zu bogeben. Oefterer Wechsel tritt ein, ja nach längerem Hinschauen scheint es fast in der Willkür zu liegen, ob man die Kugel weiter vorne neben dem liegenden Doppelbilde, oder weiter hinten unter der hängenden Kugel sehen will. Giebt es einen sichtbareren, augenfälligeren Jeweis für den Einfluss, welchen die Thätigkeit des Verstindes, oder wie man jene Seelenthätigkeit benennen mag, auf die Localisation der Doppelbilder und überhaupt auf die Objectivirung der Gesichtseindrücke ausübt?

Ehe ich diese Versuche verlasse, versäume ich nicht hinzuzufügen, dass ich, um sicher zu sein, dass es sich bei mir nicht um individuelle Eigenthümlichkeiten handle, 
wie sie bei manchen Erscheinungen des binocularen Sehens bisweilen vorkommen, jeden Versuch von anderen Individuen habe wiederholen lassen, von deren Beobachtungsfähigkeit ich mich überzengt hatte, und dass ich durchaus übereinstimmende Angaben erhielt, die von den meinen in keinem wesentlichen Punkte abwichen.

Die Resultate, welche hier gewonnen sind, beziehen sich auf das Doppeltsehen im Allgemeinen, sind unabhängig davon, welches im speciellen Falle der Grund des Doppeltsehens ist. Was für das Doppeltsehen gilt, welches durch prismatische Ablenkung der Lichtstrahlen hervorgerufen wird, gilt, wie man sich durch Wiederholung der Versuche leicht überzeugen kann, in ganz gleicher Weise auch für das Doppeltsehen, das man durch mechanische Lageveränderung des Augapfels (mittelst Fingerdruck) bewirkt. Dass auch bei dem Doppeltsehen in pathologischen Fällen, bei Erkrankung der Augenmuskeln, dasselbe Verhältniss statt hat, ist schon a priori anznnehmen. Zudem hat A. Graefe es thatsächlich erwiesen und ich kann es nach eigenen Versuchen bestätigen. Bei einer Paralyse des oberen. schiefen Augenmuskels constatirte $\Lambda$. Graefe Folgendes*): 1) Mit den Höhenunterschieden der Doppelbilder wachsen die Distancen, un welche das eine Bild näher zu stehon scheint als das andere. 2) Gleicht man die Höhenunterschiede der Doppelbilder durch Prismen aus, so rücken beide gleichzeitig in eine und dieselbe, mit der Gesichtsfläche parallele Ebene. (Aus dieser Ausdrucksweise Graefe's mag man zugleich einen Beleg für die Richtigkeit meiner obigen Behauptung entnehmen, dass die Pathologen die

*) Archir f. Ophthalm. VII. 2. pag. 111. 
ungleiche Entfernung der Doppelbilder nach dem Heraustreten des einen Doppelbildes aus der mit der Gesichtsfläche parallelen Ebene zu beurtheilen pflegen, - nicht nach dem direkten Abstand vom Auge.) "Kehrt man auf demselben Wege den Modus der Stellung um (durch ein starkes, mit der Basis vertical nach unten vor das kranke Auge zu legendes Prisma), so dass das Doppelbild, welches dem mit der Affection behafteten Auge zugehört, jetzt das höher stehende wird, so ist es damit zugleich das entfernter liegende geworden." Zum Ueberfluss hat A. Graefe ferner noch gefunden, dass für das Doppeltsehen bei Oculomotoriuslähmung dasselbe Gesetz gilt, welches bei Trochlearisparalyse das Näherstehen des einen Bildes bestimmt, während die 'Theorie von der Ectopie des Drehpunktes ein entgegengesetztes Verhalten hätte erwarten lassen. Hiernach scheint es ganz klar, dass das Näherstehen der tieferen Doppelbilder nichts specifisch Pathologisches ist, wofür ein besonderer Grund gesucht werden müsste, denn dasselbe Verhalten der in verschiedencr Höhe gesehenen Doppelbilder findet sich gerade so unter plyysiologischen Bedingungen. Die Resultate der oben erwähnten Druckexperimente v. Graefe's scheinen allein dagegen zu sprechen. Ich kann jedoch einiges Misstrauen in die Beweiskraft dieser Beobarchtungen um so weniger unterdrücken, als es mir in mehreren Fällen nicht hat gelingen wollen, bei diesen schwer auszuführenden Versuchen den gleichen Lrfolg zu erzielen. Auch bei gesunden Augen müssten ganz analoge Resultate gewonnen werden, da ja v. Graefe den Tiefenunterschied der Doppelbilder bei Trochlearislähmung durch Druck auf das gesunde Auge verändert zu haben behauptet, bei meinen eigenen Augen waren jedoch alle Bemühungen vergeblich. v. Graefe war von den Angaben seiner Kranken ablängig und dass deren Aussagen in Sachen des Doppeltsehens nicht immer zuverArchiv für Ophthalmologie. VIII, 2. 
lässig sind, weiss Jeder, der solche Untersuchungen angestellt hat. Auch wird man nach obigen Ausführungen über die Variabilität der Localisirung der Doppelbilder einige Inconstanz der Frschiciungen erklärlich finden. Jedenfalls werden erst erneute, mit allen Cautelen wiederholt angestellte Druckversuche erforderlich sein, um obiger Deduction mit Fug entgegengestellt werden $z u$ können.

Um nun noch einmal auf dic vorhin ausführlich gegebene Foerster'sche Erklärung zurückzukommen, so geht aus den angestellten Versuchen und den daran geknüpften Frörterungen zur Genüge hervor, dass dieselbe unrichtig ist. Aus eincr Reihe eng zusammenhängender Thatsachen hat nun eine vereinzelte dazu gedient, eine Erklärung zu begrüuden, die auf die anderen Thatsachen nicht mehr passt. "Wir sind gewöhnt, den Gegenstand für näher zu halten, der sich oberhalb der Macula lutea abbildet." Dies schliesst Foerster daraus, dass beim Blick auf eine in bestimmter Stellung befindliche horizoutale Flächc die derselben angehörigen Punkte, welche zwischen uns und dem in der Fläche liegenden lixirpunkt liegen, uns also näher sind als dieser, sich auf der oberen Netzhauthälfte abbilden. Dass wir aber keineswess hicran gewöhnt sind, dass jene horizontale Fläche nicht in Geringsten maassgebend für die sonstigen Verhältnisse des Sehens ist, dafür finden sich unter den obigen Versuchen mehrfache Belege. Bei Versuch IV-VI werden Bilder, welche der oberen Netzhautlıalfte angehören, ferner gesehen; auch sind die Gründe, weshalb ein Doppelbild bald näher bald ferner erscheint, als ganz andere erkannt worden.

Ebenso wenig kann eine andere Behauptung Foerster's gebilligt werden, die auf einer gleich einseitigen Schlussfolgerung beruht. „Wir sehen denselben Gegenstand mit dem oberen Theil der Netzhaut etwas kleiner, 
mit dem unteren etwas grösser." Aus dem Zusammenhange scheint hervorzugehen, dass dies als ein allgemeines Gesetz gelten soll. "Dies lässt sich auch noch auf andere Weise darthun," fügt Foerster hinzu. So lange dies nicht geschehen, muss die Richtigkeit jener Behauptung durchaus in Abrede gestellt worden. Abgesehen davon, dass die wahrgenommene Grösse des Objects, da sie lediglich von der Netzhautbildgrösse und der Entfernung abhängig ist, keinem anderweiten Gesetze folgen kann, abgesehen ferner davon, dass die der Behauptung zu Grunde liegende factische Voraussetzung unrichtig ist, so ist jener Satz schon deshalb falsch, weil er mit den bisher festgestellten Thatsachen in directem Widerspruch steht. 